\title{
Coexistence of Adenosine Deaminase, Histidine Decarboxylase, and Glutamate Decarboxylase in Hypothalamic Neurons of the Rat ${ }^{1}$
}

\author{
E. SENBA, P. E. DADDONA, ${ }^{2}$ T. WATANABE, ${ }^{3}$ J. $-Y$. WU, ${ }^{4}$ AND J. I. NAGY ${ }^{5}$ \\ Department of Physiology, Faculty of Medicine, University of Manitoba, Winnipeg, Manitoba, Canada R3E OW3
}

\begin{abstract}
Neurons immunoreactive for the enzyme adenosine deaminase (ADA) in the posterior basal hypothalamus of the rat have a distribution pattern similar to those immunoreactive for histidine decarboxylase (HDC) and are particularly numerous in the tuberal (TM), caudal (CM) and postmammillary caudal (PCM) hypothalamic magnocellular nuclei which harbor neurons containing glutamic acid decarboxylase (GAD). The extent to which these enzymes coexist within neurons of these hypothalamic regions was examined using either serial sections or simultaneous immunostaining for ADA and HDC or GAD in the same section. Analysis of serial sections revealed neuronal coexistence of ADA with HDC or GAD in both TM and CM. In addition some neurons in CM, the only area examined for triple coexistence, were found to contain all three enzymes. In sections processed for ADA simultaneously with HDC or GAD, nearly all ADA-immunoreactive neurons in TM, CM, and PCM as well as those scattered between these nuclei were found to contain HDC, and nearly all contained GAD. Exceptions to this, however, were small cells located lateral to the posterior arcuate nucleus, which appeared to contain ADA but not HDC, and large neurons located at the anterior extreme of $T M$, which appeared to contain ADA but not GAD. The relatively fow ADA-compared with GAD-containing neural systems in brain, together with the presence of ADA in GAD-containing hypothalamic magnocellular neurons, which appear to have widespread projections throughout the brain, indicate that ADA may be a convenient immunohistochemical marker for anatomical investigations of these projections. In addition, the coexistence of ADA with HDC or GAD and the probable coexistence of all three enzymes in vast majority of the dispersed neuronal
\end{abstract}

Received February 22, 1985; Revised May 6, 1985;

Accepted June 4,1985

\footnotetext{
1 This work was supported by grants from the Manitoba Health Research Council, the Medical Faculty Fund, and the Medical Research Council of Canada (MRCC) to J. I. N. who is a Scholar of the MRCC. We wish to thank M. Whittaker for skillful technical assistance and L. Polson for typing the manuscript.

${ }^{2}$ Present address: Department of Internal Medicine, University of Michigan Medical School, Ann Arbor, Ml 48109

${ }^{3}$ Present address: Department of Pharmacology II, Osaka University School of Medicine, 3-57, Nakanoshima 4, Kita-Ku, Osaka 530 Japan.

${ }^{4}$ Present address: Department of Physiology, Pennsylvania Statc Univer sity, Hershey Medical Center, Hershey, PA 17033.

${ }^{5}$ To whom correspondence should be addressed, at Department of Physiology, Faculty of Medicine, University of Manitoba, 770 Bannatyne Avenue, Winnipeg, Manitoba, Canada R3E OW3.
}

systems constituting the posterior magnocellular nuclei indicate that these systems express multiple transmitters and that they may be anatomically and functionally related.

Numerous early anatomical studies of the hypothalamus made reference to several groups of magnocellular neurons located in its posterior basal region. However, there was considerable uncertainty surrounding the anatomical relationships of these neurons. This was reflected in the diverse terminology used to describe their location which included nucleus intercalatus, mammillaris prelateralis, tuberomammillaris, and inframammillaris (Friedemann, 1911; Krieg, 1932; Crouch, 1934; Papez and Aronson, 1934; Clark, 1936; Solnitzky, 1939; Christ, 1969; Crosby and Showers, 1969; Nauta and Haymaker, 1969). Recent work by Bleier et al. (1979) represents the first detailed morphological and cytoarchitectonic characterization of the magnocellular neurons which appear to correspond to those described by earlier authors. Bleier et al. (1979) recognized that these neurons had a much wider distribution in the posterior hypothalamus than previously thought and were most numerous in three Irair lopographically relaled cenlers to which they assigned the now generaliy accepted names tuberal (TM), caudal (CM), and postmammillary caudal (PCM) magnocellular nuclei. These nuclei are located dorsolateral to the third ventricle and mammillary recess (TM), ventrolateral at the edge of the basal hypothalamus just rostral to the mammillary nucleus (CM), and ventrolateral to the mammillary peduncle just lateral to the mammillary nuclei (PCM).

The morphological resemblance of neurons in these posterior magnocellular cell groups to those located in the more rostral supraoptic and paraventricular nuclei prompted the suggestion that at least TM and $\mathrm{CM}$ may be functionally related to hypothalamic neurosecretory systems (Bleier et al., 1979). However, histochemical studies have shown that the posteriorly located neurons do not have certain features common to neurons in the supraoptic or paraventricular nuclei; they do not appear to contain neuropeptides such as vasopressin, and oxytocin. In contrast, and as suspected by Bleicr et al. (1979), there are substantial biochemical and anatomical similarities among neurons in TM, CM, and PCM as well as those in regions bridging these nuclei. Immunohistochemical studies have shown that neurons in these regions contain histamine (Wilcox and Seybold, 1982; Panula et al., 1984) and the synthetic enzyme for this putative neurotransmitter agent, histidine decarboxylase (HDC) (Watanabe et al., 1983, 1984). In similar studies, Vincent et al. (1982) reported that, although neurons containing glutamic acid decarboxylase (GAD), the marker enzyme for GABAergic systems, are found widespread in the hypothalamus, they are particularly numerous in $\mathrm{TM}, \mathrm{CM}$ and PCM. More recently, we have shown that these neuronal systems contain adenosine deaminase (ADA) (Nagy et al., 1984), the enzyme responsible for the conversion of adenosine to inosine. With respect to anatomical similarities, it has been shown that 
neurons containing either GAD, I IDC, or ADA in these regions have widespread projections to diverse brain areas including, among other structures, the cortex, striatum, and amygdala (Vincent et al., 1983; Nagy et al., 1984; Takeda et al, 1984).

The above findings, together with the similar distribution pattern of neurons containing ADA, HDC, or GAD in the posterior basal hypothalamus (Nagy et al., 1984; Watanabe et al., 1984), suggest the possibility that these enzymes coexist in hypothalamic neurons. The presence of GAD or HDC in these neurons suggests their utilization of GABA or histamine, respectively, as neurotransmitters. Although their requirement for high lovels of ADA is presently unclear, we have previously proposed that this enzyme may have some relationship to the putative neuromodulatory actions of adenosine in brain (Nagy et al., 1984, 1985). To investigate further the role of ADA and the possible involvement of multiple transmitters in posterior hypothalamic magnocellular neurons, we examined whether ADA coexists with GAD and/or HDC in these neurons and whether all three enzymes are expressed by the same neurons.

\section{Materials and Methods}

\section{Antisera and Tissue Preparation}

Antisera to purified ADA from calf intestine (Daddona and Kelley, 1977), HDC, from fetal rat liver (Taguchi et al., 1984), and GAD from mouse brain (Wu et al., 1973) were raised in rabbits and characterized with respect to specificities for and cross-reactivities with their respective antigens in rats as previously described (Wu, 1976; Nagy et al., 1984; Taguchi et al., 1984).

Male Sprague-Dawley rats weighing 200 to $300 \mathrm{gm}$ were used in the present studies. Some rats were treated $24 \mathrm{hr}$ before sacrifice with colchicine ( $50 \mu \mathrm{g} / 100 \mathrm{gm}$ of body weight) which was injected into the third ventricle while animals were maintained under Equithesin anesthesia and held in a stereotaxic apparatus. Animals were deeply anesthetized with chloral hydrate and perfused transcardially with $40 \mathrm{ml}$ of ice-cold $0.9 \%(\mathrm{w} / \mathrm{V})$ saline containing $0.1 \mathrm{M}$ sodium phosphate buffer $(\mathrm{pH} 7.4), 0.1 \%(\mathrm{w} / \mathrm{v})$ sodium nitrite, and 100 units of heparin followed by $400 \mathrm{ml}$ of an ice-cold solution containing $2.0 \%$ paraformaldehyde, $0.2 \%$ picric acid, and $0.1 \mathrm{M}$ sodium phosphate buffer $(\mathrm{pH}$ 7.4). Whole brains were removed, post-fixed in the same fixative overnight, and then transferred to $0.1 \mathrm{~m}$ phosphate buffer containing $30 \%$ sucrose for another 24 or $48 \mathrm{hr}$.

\section{Coexistence studies}

Two different methods, both involving immunohistochemical procedures, were used to examine ADA, HDC, and GAD coexistence in posterior hypothalamic magnocellular neurons. In the first, adjacent serial sections were processed separately using primary antisera to different enzymes. In the second, individual sections were processed sequentially with primary antisera to two of these three enzymes. Double immunostaining was desirable in order to obtain more reliable estimates of the extent of enzyme coexistence in the neuronal populations under investigation than that afforded by studies involving analysis of serial sections. Thus, the results derived from serial sections, although in themselves definitive, served partly as controls for this second method.

Serial sections. Transverse sections of $5 \mu \mathrm{m}$ thickness were cut on a cryostat and collected on gelatin-coated slides to yield two or three sets of serial sections. Each set consisled of two or three slides containing six to eight sections per slide with alternate sections being collected on separate slides. These sections were processed for immunohistochemistry using the peroxidase-antiperoxidase (PAP) method (Sternberger et al., 1970). One slide of sections per set was incubated with anti-ADA serum (1:500 dilution), and another was incubated with either anti-HDC serum (1:400 dilution) or with anti-GAD serum (1:500 dilution). If a third slide of sections was collected in a set, all three primary antisera were utilized, allowing analysis of up to three sets of eight triplets of consecutive sections. All primary, secondary, and tertiary antisera were diluted in a solution containing $0.9 \%$ saline, $0.1 \mathrm{M}$ sodium phosphate buffer $(\mathrm{pH} 7.4)$, and $0.6 \%$ Triton $\mathrm{X}-100$. Following incubations with primary antisera for $18 \mathrm{hr}$ at $1^{\circ} \mathrm{C}$, the sections were washed in phosphate-buffered saline (PBS: $0.1 \mathrm{~m}$ sodium phosphate buffer and $0.9 \%$ saline, $\mathrm{pH} \mathrm{7.4)}$ for $45 \mathrm{~min}$ at room temperature and incubated for $45 \mathrm{~min}$ at $37^{\circ} \mathrm{C}$ with goat anti-rabbit IgG serum (Sternberger and Myer) diluted $1: 10$. They were then washed in PBS for $45 \mathrm{~min}$, incubated for $45 \mathrm{~min}$ at $37^{\circ} \mathrm{C}$ with PAP (Sternberger and Myer) diluted 1:100, and washed in $50 \mathrm{~mm}$ Tris$\mathrm{HCl}$ butter $(\mathrm{pH}$ /.4). I he sections were then reacted with $0.02 \%$ diaminobenzidine (DAB) and $0.005 \% \mathrm{H}_{2} \mathrm{O}_{2}$ in Tris-HCl buffer for 10 to $20 \mathrm{~min}$, dehydrated, coverslipped with Permount, and photographed using interference contrast optics.

Analysis of enzyme coexistence in neurons was conductd with the aid of acetate transparencies. Immunoreactive neurons and landmarks such as blood vessels and section edges on photomicrographs of one set of a pair of adjacent sections were traced onto transparencies, and these were overlayed onto those of adjacent sections. Coexistence was considered inconclusive unless there was an exact correspondence of at least three immunoreactive neurons and several landmarks on adjacent sections. This criterion was found to largely exclude false positives, whereas a less stringent one led to uncertainty with respect to the identification of corresponding neurons

Simultaneous immunostaining for two antigens. Utilization of indirect immunofluorescent methods with different second antibody fluorochromes was not possible since all three primary antisera employed in this study were prepared in the same species. Consequently, three other double immunostaining procedures were tried: (1) immunofluorescent staining with a first primary antibody followed by elution and immunofluorescent staining with a different second primary antibody, (2) dual color immunostaining using the PAP method where one antigen is first revealed by the brown reaction product of DAB and the second by the blue reaction product of 4-chloro- 1 . naphthol $(\mathrm{CN})$, and (3) immunostaining for one antigen using the PAP method followed by immunofluorescent staining for a second antigen. These procedures have previously been described by Tramu et al. (1978), Sternberger and Joseph (1979), and Belin et al. (1983), respectively.

The first two of these methods proves unsuccessful in the present system. In the first method, either the primary sequence antibody failed to elute completely, as indicated by control procedures, or the second sequence antibody failed to produce immunostaining, probably as a result of antigen denaturation. The second and third methods, as used here, both rely on the finding by Sternberger and Joseph (1979) that, under appropriate conditions, deposition of $D A B$ reaction product in the unlabeled antibody method eliminates peroxidase activity of the PAP complex and antigenicity of the immunoreagents involved, thereby allowing immunohistochemical reprocessing of sections for a second antigen without interference by that of the first. In principle, therefore, both of these methods were expected to be feasible. However, with respect to the second method, although both DAB and CN when employed alone as substrates for PAP produced reliable immunostaining, it became difficult to distinguish blue over brown reaction product when $D A B$ and $C N$ were used in the first and second sequence reactions, respectively. This was true even when low concentrations of primary antiserum were employed in the first sequence reaction. However, neurons containing both colors were evident in some cases, as were cells containing only blue after processing of sections for two antigens. Finally, in the third method, we found, in contrast to the results of Belin et al. (1983), that the PAP reaction product from the first sequence reaction tonded to obscuro the second sequence immunofluorescent staining. Thus, this method may be generally impractical for the visualization of two different antigens having similar subcellular distributions. Fortunately, two of the antigeris under irivestigation here, namely HDC and GAD, are restricted to neuronal cytoplasm, whereas ADA is present in both the cytoplasm and nucleus. Therefore, simultaneous immunostaining for two antigens was conducted as follows.

Sections $(15$ to $20 \mu \mathrm{m})$ were obtained on a sliding microtome and processed free floating for HDC or GAD immunohistochemistry using the PAP method with DAB substrate as described above. Following the DAB reaction the sections were washed in PBS, incubated with anti-ADA serum for $48 \mathrm{hr}$ at $4^{\circ} \mathrm{C}$, washed in PBS for $45 \mathrm{~min}$, and incubated with fluorescein isothiocyanate (FITC)-conjugated sheep anti-rabbit serum (Statens Bakteriologiska, diluted $1: 10$ ) for $45 \mathrm{~min}$ at $37^{\circ} \mathrm{C}$. Following a final wash in PBS for 45 min, the sections werc mounted onto gelatinized slides, coverslipped with glycerol:water (1:1), and photographed by both brightfield and fluorescence microscopy for visualization of DAB reaction product or FITC in neuronal cytoplasin or muclei, respeclively. Control procedures included processing of tissues exactly as described above but with the omission of the step involving incubation with anti-ADA serum.

\section{Results}

\section{Distribution and appearance of immunoreactive neurons}

Immunostaining for ADA was intense in neuronal cell bodies including their dendrites and nuclei. ADA-immunoreactive neurons densely packed in the core regions of the TM, CM, and PCM were medium to large in size (15 to $30 \mu \mathrm{m}$ ) and round or oval in shape. Most, though not all, of those scattered outside these centers tended 
to be smaller and more fusiform or triangular. A more detailed description of the anatomical arrangement of these hypothalamic ADA-containing neurons will be reported clscwhore (J. I. Nagy and E. Senba, manuscript in preparation). After colchicine treatment, there was no apparent increase in the number of ADA-immunoreactive cells although immunostaining was slightly intensified.

Immunostaining for HDC was found in neuronal cytoplasm and dendritic processes, but not in neuronal nuclei. As in the case of ADA-immunoreactive neurons, HDC-positive cells were medium to large $(15$ to $30 \mu \mathrm{m})$ and mostly round or oval within the posterior hypothalamic magnocellular nuclei (PHMN) proper, whereas outside these nuclei they were smaller and appeared fusiform or triangular. The distribution of neurons containing HDC was similar to those containing ADA. Interestingly, although the distribution pattern of HDC-immunoreactive neurons was qualitatively unaltered in animals pretreated with colchicine, HDC-immunostaining was substantially reduced such that light staining was observed only in neuronal soma and not in dendritic processes. This result was obtained whether progressively higher or lower dilutions of primary antiserum were used.

In the absence of colchicine pretreatment, GAD-immunoreactive perikarya remained elusive although numerous punctate deposits of $\mathrm{DAB}$ reaction product, presumed to represent GAD-containing terminals, were seen in the posterior hypothalamus as well as throughout the brain. Colchicine pretreatment decreased punctate immunoslaining and concomitantly revealed GAD-containing cell bodies in which DAB reaction product was restricted to neuronal cytoplasm and appeared granular. Neurons immunoreactive for GAD were found widely distributed in the posterior hypothalamic area as well as concentrated in discrete nuclei consisting of heterogenously sized cells. Small- to medium-sized fusiform or triangular neurons with long immunostained processes (10 to $15 \mu \mathrm{m}$ ) were scattered in the area surrounding the fornix and mammillothalamic tract. Considerable numbers of small $(<10 \mu \mathrm{m})$ GAD-positive neurons were observed in the zona incerta, the supramammillary nucleus, and the periventricular area just beneath TM extending caudally to the arcuate nucleus, within which they were found situated medially. Medium- to large-sized GAD-containing (15 to $30 \mu \mathrm{m}$ ) neurons, mostly round or oval in shape, were observed in the TM, CM and PCM, with a distribution similar to those containing ADA and HDC.

\section{Neuronal enzyme coexistence}

Serial sections. Since neurons containing ADA, HDC, or GAD in the hypothalamus were most numerous within TM or $C M$ and in immediately surrounding arcas, investigations of the coexistence of these enzymes using serial sections were restricted to these nuclei. In photomicrographs of more than 30 pairs of adjacent sections through TM and $C M$, many neurons were found to be immunoreactive for both ADA and HDC (Fig. 1). In the core areas of these nuclei, particularly $\mathrm{CM}$, immunoreactive cells were so closely packed and intensely stained that it was sometimes difficult to identify single neurons and, therefore, the same neuron in adjacent sections (Fig. $1, e$ and $f$ ). However, in regions just peripheral to these nuclei, where cells were more dispersed, portions of the same neurons which could be positively identified to be present in adjacent sections were always found to be immunoreactive for both ADA and $\mathrm{HDC}$. In addition, whenever corresponding neurons could be identified within the dense cluster of cells in CM, coexistence of these enzymes was invariably found. Examples of neurons containing both enzymes are shown in adjacent sections (Fig. 1) through TM (Fig. 1, a and b), the rostral part of the CM (Fig. 1, $C$ and $d$ ), and CM (Fig. 1,e and $f$ ).

Similar results were obtained in studies involving ADA and GAD where, again, more than 30 pairs of adjacent sections through TM and $C M$ were analyzed. All immunostained neurons which could be identified as portions of the same neuron in adjacent sections were immunoreactive for both of these enzymes (Fig. 2). This was true of neurons dispersed in the periphery of TM and $\mathrm{CM}$ as well as those within CM.

The presence of ADA, HDC, and GAD in individual hypothalamic neurons was examined in more than 20 triplets of photomicrographs of serial sections through CM only. In each of these triplets, an example of which is shown in Figure 3, several neurons were found to contain all three enzymes. Although immunostaining for $\mathrm{HDC}$ was reduced in these experiments by virtue of the colchicine pretreatment required to visualize GAD, HDC-immunoreactive neurons were still sufficiently recognizable to allow comparisons with GAD- and ADAcontaining neurons in adjacent sections.

It should be noted here that the above analysis, despite the use of relatively thin serial sections, tends to be biased toward revealing coexistence among large neurons; individual small neurons will appear less often in both of a pair of adjacent sections. In addition, the adjacent sections examined here were not counterstained since this would have compounded the difficulty of identifying corresponding neurons in the densely packed cell groups of TM and CM. As a consequence, the above results provide no information concerning possible lack of coexistence since we could not determine the frequency with which individual immunoreactive neurons appeared in only one of a pair of consecutive sections. Thus, in these experiments it was not possible to obtain a false negative result, i.e., an erroneous lack of coexistence, since only neurons immunoreactive for the enzymes under investigation were visualized and only those exhibiting enzyme coexistence were recognized as corresponding in adjacent sections.

Simultaneous immunostaining for two enzymes. Analysis of sections simultaneously immunostained for either HDC or GAD by the PAP method and for ADA by the indirect immunofluorescent method was best conducted microscopically prior to photography. Sections could be viewed either by alternating between illumination for brightfield and fluorescence or by simultaneous illumination for both, with that of brightfield being kept relatively low. Either way neurons exhibiting both cytoplasmic DAB reaction product and nuclear fluorescence could be detected without difficulty. Occasionally, ADA immunofluorescence could be detected throughout the soma of neurons lightly immunostained for HDC or GAD. Conversely, nuclear immunofluorescence tended to be obscured in cases where neurons contained dense cytoplasmic DAB reaction product overlying the nucleus. However, although it cannot be appreciated in the black and white photomicrographs shown here, faint green nuclear immunofluorescence could still be detected in most of these cases.

Virtually all ADA-immunoreactive neurons in the hypothalamus were also immunoreactive for HDC (Fig. 4). This included large and small neurons in TM (Fig. 4, $a$ and b), CM (Fig. 4, $c$ and $d$ ), and PCM (Fig. 4, e and $f$ ), as well as those dispersed between and connecting these nuclei (Fig. 6, $c$ and $d$ ). Similarly, virtually all neurons exhibiting ADA immunofluorescence in CM (Fig. 5d), PCM (Fig. $5 f$ ), and related regions (Fig. $6 f$ ), and nearly all of those in TM (Fig. 5b) were also immunostained for GAD (Figs. 5, $c$ and $e, 6 e$, and $5 a$, respectively). However, at the dorsal anterior extreme of TM where neuronal coexistence of ADA and HDC was observed, neurons were found which appeared to contain ADA (Fig. 6h) but not GAD (Fig. 6g). In addition, two regions were found in which ADA and $\mathrm{HDC}$ appeared to be separate. In the area flanking the posterior arcuate nucleus, several neurons per section exhibited immunofluorescent staining for ADA but were clearly devoid of HDC immunoreactivity (not shown). In contrast, small numbers of cells weakly immunoreactive for HDC within the arcuate nucleus were consistently devoid of ADA immunostaining.

In control experiments no immunofluorescent staining was observed in sections processed immunohistochemically for HDC or GAD by the PAP method (illustrated for HDC in Fig. 6a) followed by reprocessing using the immunofluorescent method but with omission of anti-ADA serum (Fig. 6b). Moreover, in sections sequentially processed for GAD, then ADA, no ADA-immunofluorescent staining was observed in the cytoplasm or nucleus of even lightly immuno- 

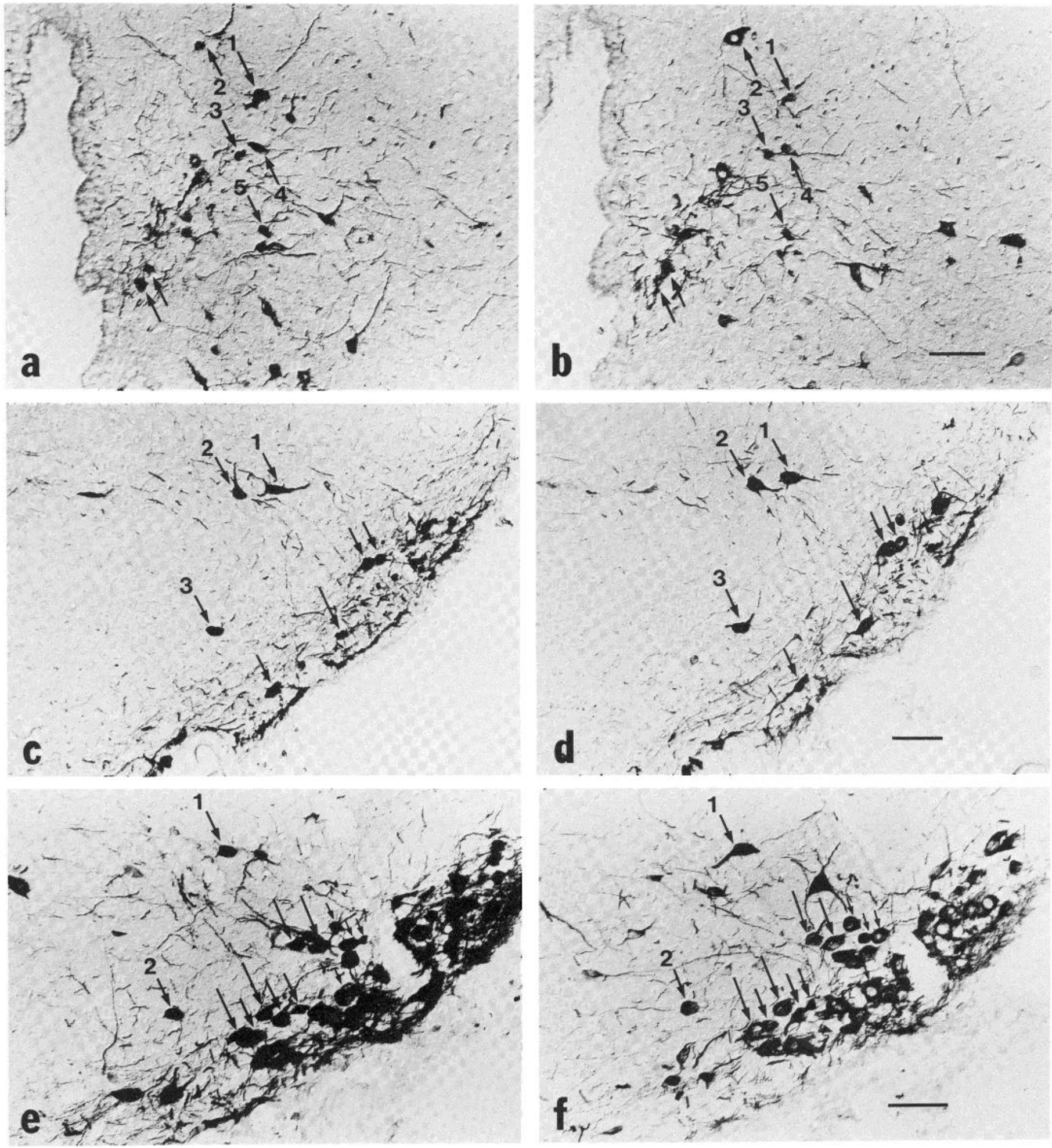

Figure 1. Photomicrographs of serial sections demonstrating coexistence of ADA and HDC in posterior hypothalamic neurons. Adjacent sections through $\operatorname{TM}(a$ and $b)$, the rostral part of $\mathrm{CM}(c$ and $d)$, and the center of $\mathrm{CM}(e$ and $f)$ show neurons immunoreactive for both ADA $(a, c$, and $e)$ and HDC $(b, d$, and $f$ ). The arrows, which in some cases are numbered to aid identification, indicate portions of the same neuron in corresponding adjacent sections. Scale bars, $50 \mu \mathrm{m}$.

stained GAD-containing neurons outside the hypothalamus, indicating that the second sequence immunoreagents did not cross-react with those of the first which were presumably blocked by the DAB reaction product. Immunostaining for ADA was abolished by pread- sorption of antiserum to this enzyme with purified ADA. Immunostaining for GAD or HDC within or outside the hypothalamus was not altered by preadsorption of antiserum to these enzymes with ADA. That antisera to ADA, GAD, and HDC do not cross-react with 

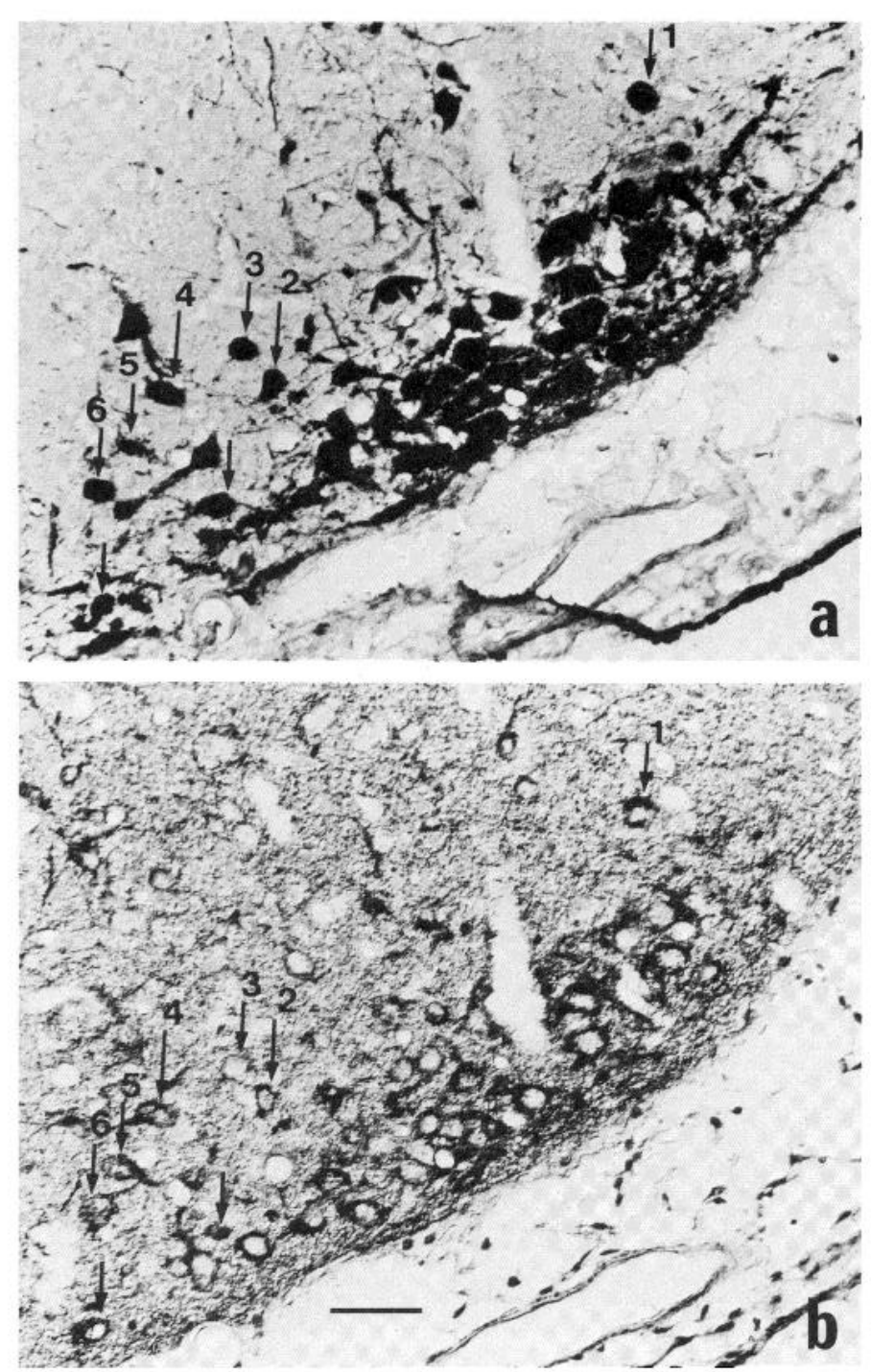

Figure 2. Photomicrographs of adjacent sections showing coexistence of ADA (a) and GAD (b) in neurons of CM. Arrows indicate corresponding neurons immunoreactive for both enzymes. Scale bar, $50 \mu \mathrm{m}$.

each of the other's antigen is further indicated by: (1) the requirement of cholchicine pretreatment to visualize GAD but not the other two enzymes in hypothalamic neurons, (2) the failure to immunostain ADA- or GAD-containing structures outside the hypothalamus with antiserum to $\mathrm{HDC},(3)$ the visualization of distinct and different fiber tracts with antiserum to either ADA or HDC but failure to visualize these same tracts with antiserum to GAD, and (4) the failure of antisera to GAD or HDC to produce nuclear immunostaining of hypothalamic neurons which clearly exhibit such staining with antiserum to ADA.

\section{Discussion}

Although the detailed anatomy of the neuronal system constituting the PHMN has been largely ignored until recently, this system is now likely to attract, and indeed, warrants, considerable attention in view of its various and potentially important biochemical and anatomical features. As previously shown and confirmed here, the three major centers comprising the PHMN, namely $\mathrm{TM}, \mathrm{CM}$, and $\mathrm{PCM}$, and the regions between these centers, all harbor an array of neurons immunoreactive for ADA, GAD, or HDC (Vincent et al., 1982; Nagy et al., 1984; Watanabe et al., 1984). We now further demonstrate the relationship of ADA-containing neurons in these areas to those containing GAD or HDC. Analysis of serial sections revealed that many neurons immunoreactive for $A D A$ also contained $\mathrm{HDC}$ or GAD. Moreover, double immunostaining procedures showed
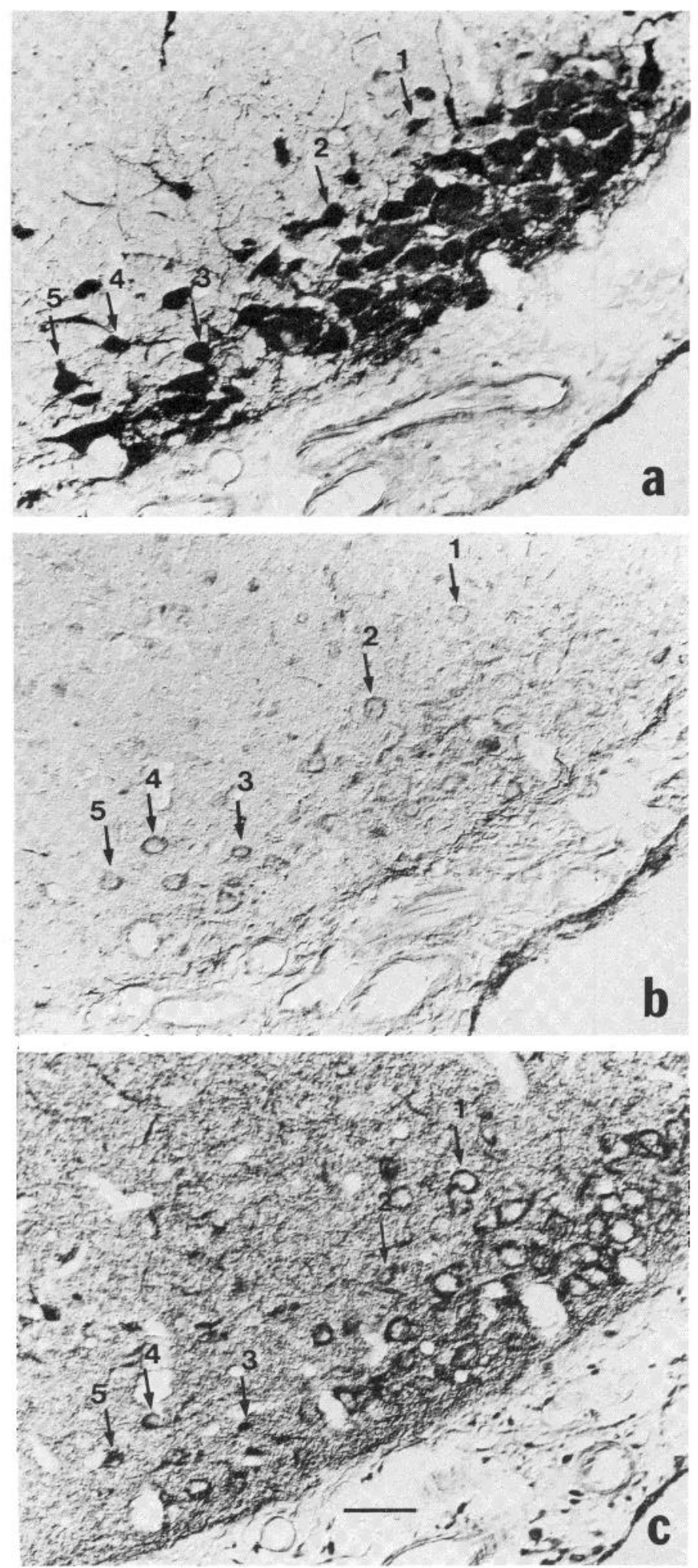

Figure 3. Photomicrographs of three serial sections showing neurons immunoreactive for ADA (a), HDC (b), and GAD (c) in CM. The numbered arrows indicate portions of the same neurons which appear in all three micrographs and which are immunoreactive for all three enzymes. Scale bar, $50 \mu \mathrm{m}$. 

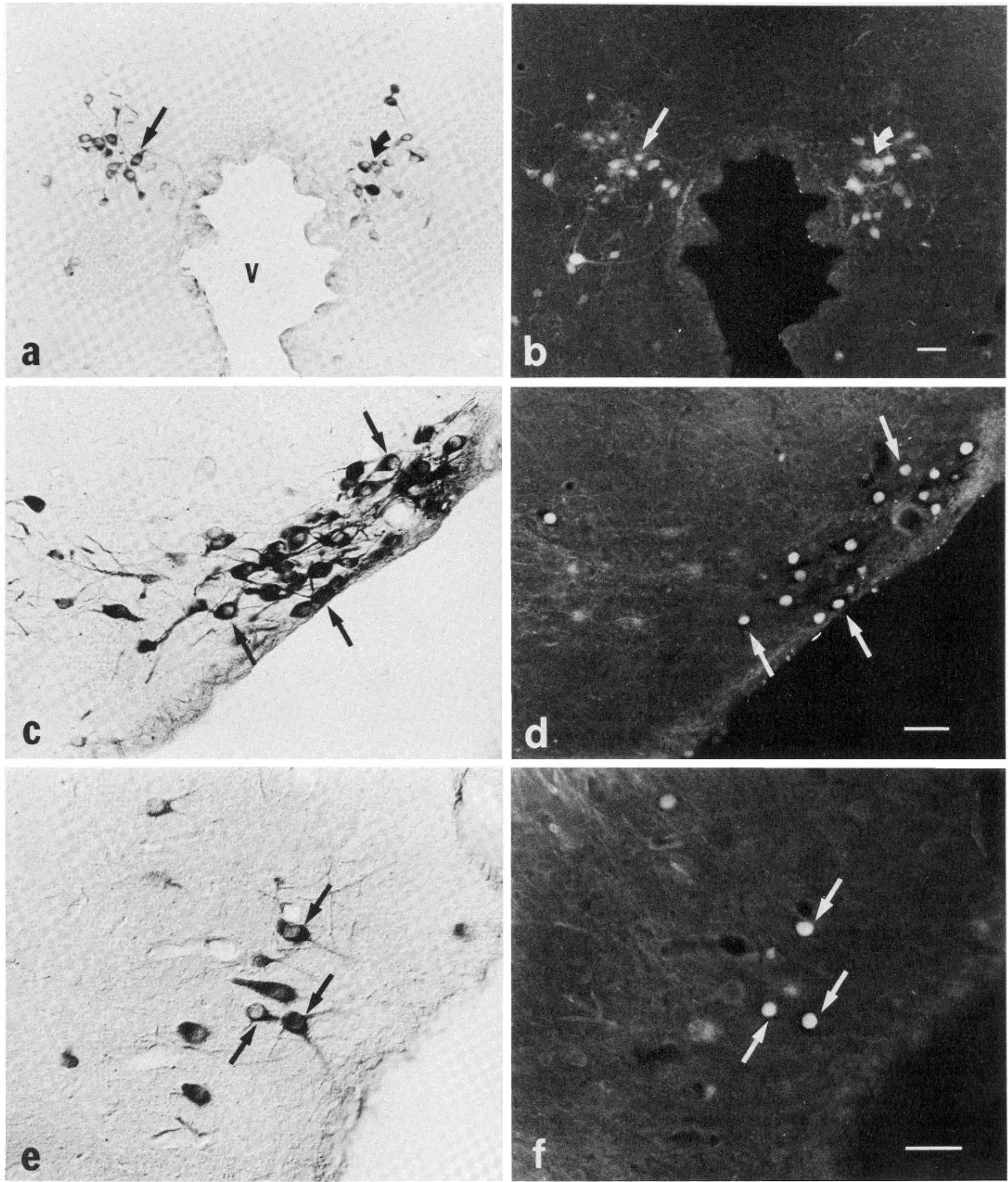

Figure 4. Simultaneous immunostaining for $\operatorname{HDC}(a, c$, and $e$; brightfield micrographs) and $\operatorname{ADA}(b, d$, and $f$; immunofluorescent micrographs) in neurons of PHMN. Sections were processed first for HDC by the PAP method and then for ADA by the immunofluorescence method. Straight and curved arrows indicate examples of individual neurons or groups of neurons, respectively, that are immunoreactive for both HDC and ADA in the same section through TM $(a$ and $b), C M(c$ and $d)$, and PCM ( $e$ and $f$ ). Although not evident here, virtually all HDC-positive neurons were seen to exhibit some green (FITC) immunofluorescence. $V$, third ventricle. Scale bars, $50 \mu \mathrm{m}$. 

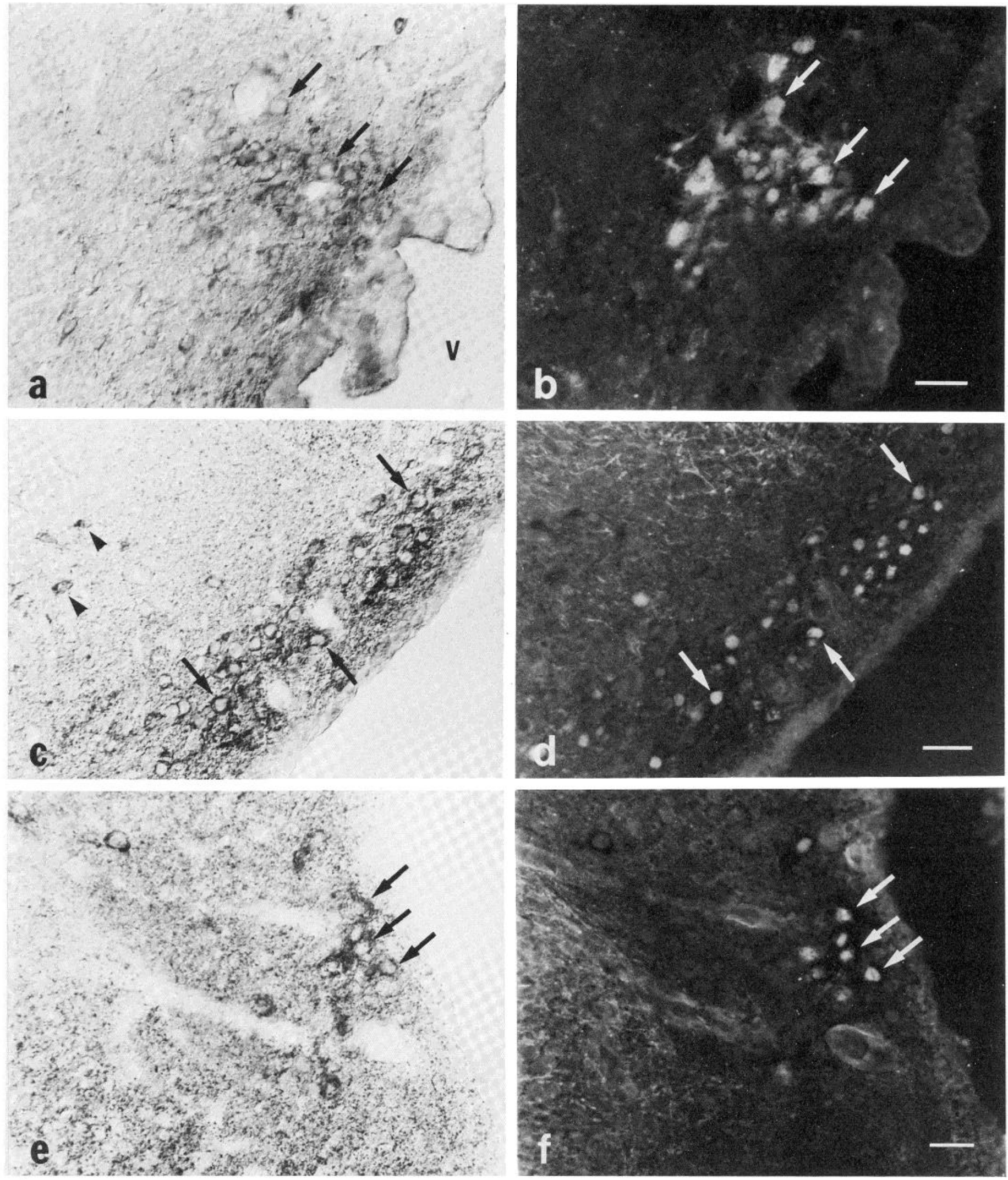

Figure 5. Simultaneous immunostaining for $\operatorname{GAD}(a, c$, and $e)$ and $\operatorname{ADA}(b, d$, and $f$ ) in neurons of PHMN. Sections were processed first for GAD by the PAP method and then for ADA by the immunofluorescence method and photographed using brightfield (GAD) or fluorescence (ADA) microscopy. Arrows indicate examples of neurons immunoreactive for both GAD and ADA in the same section through TM ( $a$ and $b)$, CM ( $c$ and $d)$, and PCM (e and $f)$. Virtually all ADA-positive neurons, including some in which green immunofluorescence is not evident in these micrographs, were immunoreactive for GAD. However, some GAD-positive neurons were devoid of ADA-immunostaining (c, arrowheads). $V$, third ventricle. Scale bar, $50 \mu \mathrm{m}$. 

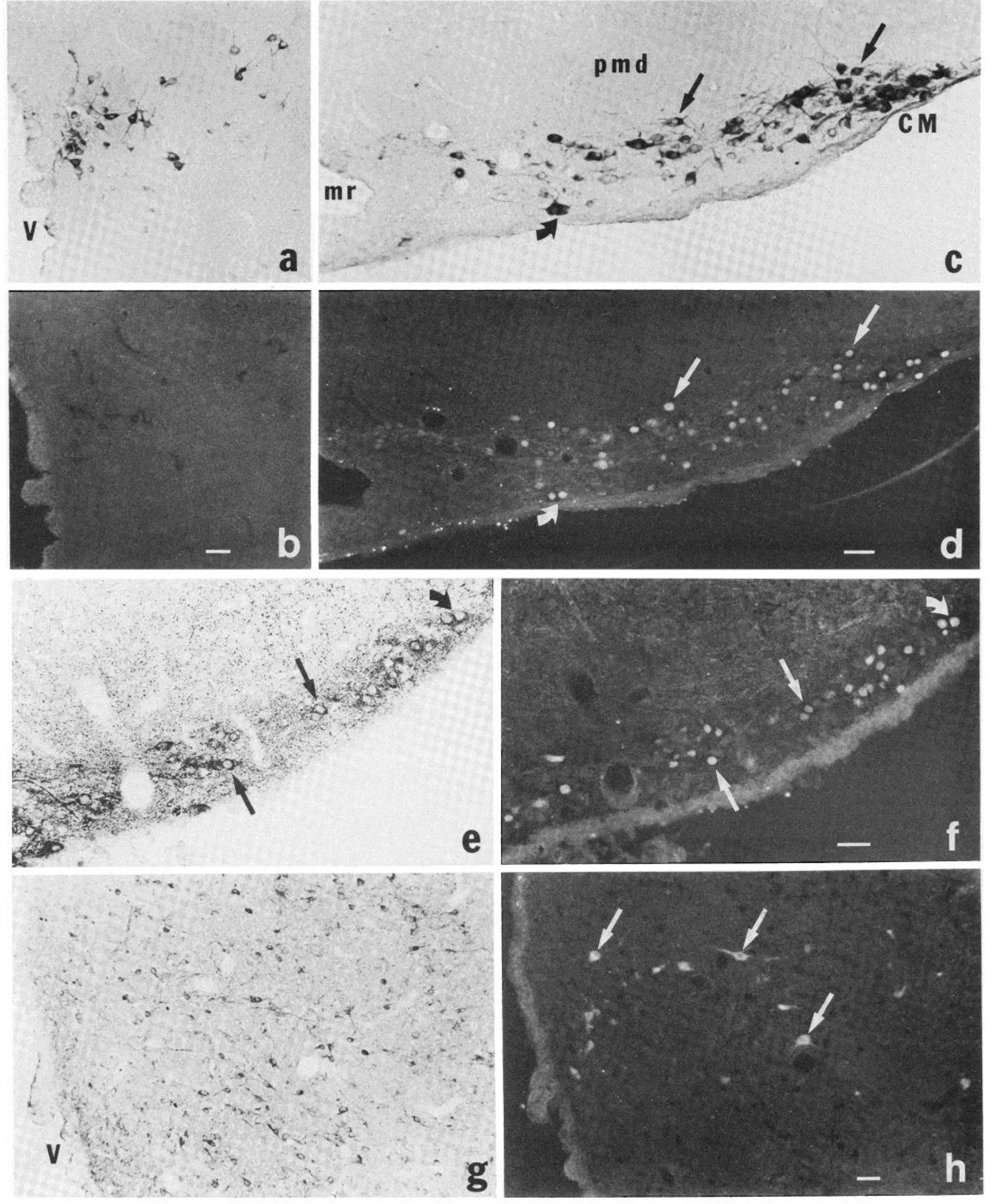

Figure 6. Simultaneous immunostaining for ADA and HDC or GAD in sections through the posterior hypothalamus. Sections were processed first for either HDC or GAD by the PAP method and then for ADA by the indirect immunofluorescence method and photographed using brightfield (HDC or GAD) or immunofluorescence (ADA) microscopy, $a$ and $b$, Micrographs of the same section showing that, after processing of tissue for HDC (a), no 
that the neuronal coexistence of ADA with either of the two decarboxylase enzymes was widespread, encompassing nearly all areas of the PHMN. The extent to which all three enzymes coexist in the same neurons is presently unclear. However, certain recent observations may have some bearing on this point. It has been reported that nearly all of the large neurons in TM, CM, and PCM contain GAD (Vincent et al., 1983) and that nearly all of these neurons are immunoreactive for both GAD and HDC (Takeda et al., 1985). On this basis, ADA is likely to be present in substantial numbers of neurons containing both HDC and GAD. This is consistent with examples shown here of individual neurons containing all three enzymes. This conclusion is further supported by the diverse but often similar brain areas to which ADA-, GAD-, and HDC-containing hypothalamic neurons have projections. These include the striatum, cortex, and amygdala (Vincent et al., 1983; Nagy et al., 1984; Takeda et al., 1984). A more delailed comparison of the central distribution of HDC- and ADA-immunoreactive fibers indicates that wherever the former are found, so too are the latter. The converse, however, is not true since, unlike HDC-positive perikarya which are found only in the hypothalamus, ADA-positive neurons are present in several other brain regions (J. I. Nagy and E. Senba, in preparation).

Although the above results indicate substantial coexistence of ADA with HDC or GAD in PHMN, we cannot exclude the possibility of some lack of coexistence. A few large neurons 6 to 10 per section) at the extreme rostral pole of TM appeared to contain ADA but not GAD. This also appeared to be the case for some large neurons spanning the dorsal region between $\mathrm{CM}$ and the mammillary recess. That these results are isolated examples of false negatives in the double immunostaining experiments cannot be ruled out for two rcasons; immunohistochemical methods cannot be considered $100 \%$ reliable for revealing low levels of antigens, and in the case of studies involving GAD, colchicine treatment may have caused insufficient accumulation of this enzyme, particularly in large neuronal cell bodies. However, neurons containing ADA independently of GAD were observed in the midst of large and small neurons containing both enzymes. It may also be noteworthy that in our earlier studies some neurons devoid of ADA immunoreactivity (Nagy et al., 1984), but clearly within the PHMN, could be retrogradely labeled from the cortex or striatum with the fluorescent tracer fast blue. With respect to $A D A$ and $H D C$, in the attempts to examine the coexistence of these enzymes by using dual color immunostaining in the same section where HDC was first visualized using DAB followed by the visualization of ADA using $\mathrm{CN}$, some small cells immediately lateral to the posterior arcuate nucleus were clearly immunostained blue (CN) but were devoid of brown reaction product (DAB). Small ADApositive cells lacking HDC immunoreactivity were also found in similar locations in sections processed sequentially for HDC by the PAP method and for ADA by the indirect immunofluorescent method. In contrast, cells containing only HDC were observed in the arcuate nucleus. The possible significance of the biochemical heterogeneity observed in minor populations of neurons in $\mathrm{PHMN}$, with respect to ADA, HDC, and GAD content, may become evident through more detailed studies regarding the projections of, and transmitters utilized by, these neurons. At present we cannot exclude that HDC-positive cells in the arcuate nucleus are mast cells since there is evidence for the presence of these in the basal hypothalamus (Roberts and Calcutt, 1983).

Two issues need to be considered regarding the neurotransmitter nature of the néurons in PHMN: one concerns their axon terminals and the other their cell bodies. The anatomical findings cited above, together with studies showing that hypothalamic lesions reduce HDC activity and histamine levels in forebrain regions (Garbarg et al., 1974, 1976), indicate that HDC is transported to, and histamine is synthesized in, nerve terminals of the HDC-containing ascending neuronal system. Similarly, inasmuch as GAD is a well established marker for GABA-containing neurons, it too is probably transported and actively involved in the production of GABA in this system. The failure to reduce GAD or GABA levels in, for example, the cortex after lesions interrupting GAD-containing projections to this region (Ulmar et al., 1975) is probably due, in part, to the high intrinsic content of cortical GABAergic elements and the small contribution made to this by the hypothalamocortical neurons. Thus, GABA and/ or histamine may be utilized as transmitters and/or co-transmitters in these neurons. Whether adenosine, a substance gaining some recognition as a possible neuromodulator in the CNS (Phillis and $\mathrm{Wu}, 1981$ ), subserves such a role in these neurons, as may be represented by their high ADA content, is still a matter of speculation (Nagy et al., 1984). With respect to the cell bodies of the hypothalamic magnocellular neurons, an analogy may be drawn between these and the neurons giving rise to the diffuse central noradrenergic, serotonergic, and dopaminergic projection systems. All of the neurons of these systems contain within their cell bodies the enzymes necessary for the synthesis of their respective transmitter; these perikaryal enzymes, and, in some cases, their related transmitters can be visualized histochemically or immunohistochemically without the need for colchicine pretreatment to inhibit their axoplasmic transport out of the cell body (Moore and Bloom, 1978, 1979). There is now strong evidence that dendrites of these neurons release their respective transmitter which then acts presynaptically on autoreceptors or postsynaptically on nerve terminals (Aghajanian, 1981; Glowinski and Cheramy, 1981). It is therefore conceivable that, whatever their true neurotransmitter nature, the hypothalamic magnocellular perikarya, which also give rise to diffuse projections, express en zymes involved in transmitter metabolism, and in some cases contain vesicular-like structures (Maeda et al., 1984), may also exhibit dendritic relcase of their transmitter(s).

Other biochemical features of hypothalamic neurons distributed in a topographic configuration similar to those containing ADA, GAD, and HDC include cells that have: (1) high monoamine oxidase activity and projections to the cortex in rat and cat (Kitahama et al., 1984; Maeda et al., 1981; Tago et al., 1981); (2) immunoreactivity for thyrotropin-releasing hormone (Shirozu et al., 1985) and histamine (Panula et al., 1984); (3) the ability to take up serotonin (Chan-Palay, $1977) ;(4)$ the capacity to accumulate and decarboxylate 5-hydroxytryptophan (Sakumoto et al., 1984); and (5) high levels of binding sites for the ligand ${ }^{3} \mathrm{H}$-nitrobenzylthioinosine, a putative ligand for adenosine uptake sites in rat brain (Geiger et al., 1985; Nagy et al., 1985). Whether all of these characteristics are expressed simultaneously by individual hypothalamic neurons remains to be determined. This notwithstanding, the observation that these characteristics are displayed by a confluence of neurons conforming to the system of PHMN described by Bleier et al. (1979) lends credence to their notion that these nuclei constitute a single functionally related anatomical system.

\section{References}

Aghajanian, G. K. (1981) Regulation of central noradrenergic cell firing: Role of alpha-adrenoreceptors and opiate receptors. In Chemical Neurotrans mission. 75 Years, L. Stjarne, P. Hedquist, H. Lagercrantz, and A. Wen malm, eds., pp. 274-284, Academic Press, Inc., New York.

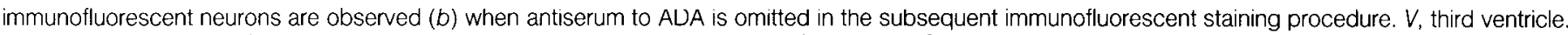

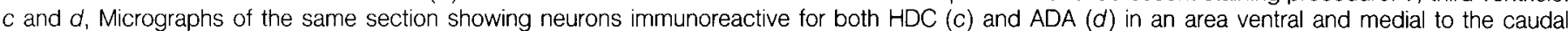

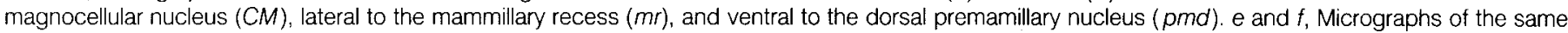

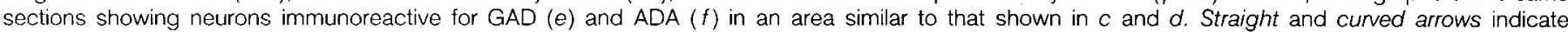

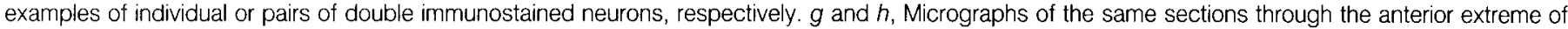
$\mathrm{TM}$ showing neurons which failed to immunostain for GAD $(g)$ but which were immunoreactive for ADA ( $h$, arrows) 
Belin, M. F., D. Nanopoulos, M. Didier, M. Aquera, H. Steinbusch, A. Verhofstad, M. Maitre, and J. F. Pujol (1983) Immunohistochemical evidence for the presence of $\gamma$-aminobutyric acid and serotonin in one nerve cell. A study on the raphe nuclei of the rat using antibodies to glutamic acid decarboxylase and serotonin. Brain Res. 275: 329-339.

Bleier, R., P. Cohn, and I. K. Siggelkow (1979) A cytoarchitectonic atlas of the hypothalamus and hypothalamic third ventricle of rat. In Handbook of the Hypothalamus. Vol. 1: Anatomy of the Hypothalamus, P. J. Morgane and J. Panksepp, eds., pp. 137-220, Marcel Dekker, Inc., New York.

Chan-Palay, V. (1977) Indoleamine neurons and their processes in normal rat brain and in chronic diet induced thiamine deficiency demonstrated by uptake of ${ }^{3} \mathrm{H}$-serotonin. J. Comp. Neurol. 176: 467-494.

Christ, J. F. (1969) Derivation and boundaries of the hypothalamus, with atlas of hypothalamic grisea. In The Hypothalamus, W. Haymaker, E. Anderson, and W. J. H. Nauta, eds., pp 13-60, Charles C Thomas, Springfield, IL.

Clark, W. E. L. (1936) The topography and homologies of the hypothalamic nuclei in man. J. Anat. 70: 203-214.

Crosby, E. C., and M. J. C. Showers (1969) Comparative anatomy of the preoptic and hypothalamic areas. In The Hypothalamus, W. Haymaker, E. Anderson, and W. J. H. Nauta, eds., pp. 61-135, Charles C Thomas, Springfield, IL.

Crouch, R. L. (1934) The nuclear configuration of the hypothalamus and thalamus of Macacus rhesus. J. Comp. Neurol. 59: 431-449.

Daddona P. E., and W. N. Kelley (1977) Human adenosine deaminase Purification and subunit structure. J. Biol. Chem. 252: 110-115.

Friedemann, M. (1911) Die cytoarchitektonik des Zwishenhirns der Cercopitheken mit besonderer Berucksichtigung des Thalamus opticus. J. Psychol. Neurol. 19: 309-378.

Garbarg, M., G. Barbin, J. Forger, and J. C. Schwartz (1974) Histaminergic pathway in rat brain evidenced by lesions of the medial forebrain bundle. Science 186: 833-834.

Garbarg, M., G. Barbin, S. Bischoff, H. Pollard, and J. C. Schwartz (1976) Dual localization of histamine in an ascending neuronal pathway and in non-neuronal cells evidenced by lesions in the lateral hypothalamic area. Brain Res, 106: 333-348.

Geiger, J. D., F. S. LaBella, and J. I. Nagy (1985) Characterization of nitrobenzylthioinosine binding to nucleoside transport sites selective for adenosine in rat brain. J. Neurosci. 5: 735-740.

Glowinski, J., and A. Cheramy (1981) Dendritic release of dopamine: Its role in the subtantia nigra. In Chemical Neurotransmission. 75 Years, L. Stjarne, P. Hedquist, H. Lagercrantz, and A. Wenmalm, eds., pp. 285-299, Academic Press, Inc., New York.

Kitahama, K., K. Sakai, H. Tago, H. Kimura, T. Maeda, and M. Jouvet (1981) Monoamine oxidase-containing neurons in the cat hypothalamus: Distribution and ascending projection to the cerebral cortex. Brain Res. 324: 155-159.

Krieg, W. J. S. (1932) The hypothalamus of the albino rat. J. Comp. Neurol. 55: $19-89$

Maeda, I., H. Kimura, T. Nagai, H. Imai, R. Arai, T. Sakumoto, K. Sakai, K Kitahama, and M. Jouvet (1984) Histochemistry of the magnocellular neurons in the posterior hypothalamus, with special reference to MAO activity and ability of 5 HTP uptake and decarboxylation. Acta Histochem. Cytochem. 17: 179-193.

Moore, R. Y., and F. E. Bloom (1978) Central catecholamine neuron systems: Anatomy and physiology of the dopamine systems. Annu. Rev. Neurosci. I: $129-169$

Moore, F. Y., and F. E. Bloom (1979) Central catecholamine neuron systems: Anatomy and physiology of the norepinephrine and epinephrine systems. Annu. Rev. Neurosci. 2: 113-168.

Nagy, J. I., L. A. LaBella, M. Buss, and P. E. Daddona (1984) Immunohistochemistry of adenosine deaminase: Implications for adenosine neurotransmission. Science 224: 166-168.

Nagy, J. I., J. D. Geiger, and P. E. Daddona (1985) Adenosine uptake sites in rat brain: Identification using ${ }^{3} \mathrm{H}$-nitrobenzylthioinosine and co-localization with adenosine deaminase. Neurosci. Lett. 55: 47-53.

Nauta, W. J. H., and W. Haymaker (1969) Hypothalamic nuclei and fiber connections. In The Hypothalamus, W. Haymaker, E. Anderson, and W. J. H. Nauta, eds., pp. 136-209. Charles C Thomas, Springfield, IL.

Panula, P., H. - Y. T. Yang, and E. Costa (1984) Histamine-containing neurons in the rat hypothalamus. Proc. Natl. Acad. Sci. U. S. A. 81: 2572-2576.
Papez, J. W., and L. R. Aronson (1934) Thalamic nuclei of Pithecus (MacaCus) rhesus. I. Ventral thalamus. Arch. Neurol. Psychol. 32: 1-26.

Phillis, J. W., and P. H. Wu (1981) The role of adenosine and its nucleotides in central synaptic transmission. Prog. Neurobiol. 16: 187-239.

Roberts, F., and C. R. Calcutt (1983) Histamine and the hypothalamus. Neuroscience 9: 721-739.

Sakumoto, T., K. Sakai, M. Jouvet, H. Kimura, and T. Maeda (1984) 5-HT immunoreactive hypothalamic neurons in rat and cat after 5-HTP administration. Brain Res. Bull. 12: 721-733.

Shirouzu, M., H. Kimura, T. Yamamoto, J. Ochi, and K. Inanaga (1985) Production of new type of antisera to thyrotropin releasing hormone (TRH) using carbodiimide coupling and an application to immunohistochemistry. Kurume Med. J. 30: 1-9.

Solinitzky, O. (1939) The hypothalamus and subthalamus of Sus scrofa. J. Comp. Neurol. 70: 191-229.

Sternberger, L. A., and S. A. Joseph (1979) The unlabelled antibody method. Contrasting color staining of paired pituitary hormones without antibody removal. J. Histochem. Cytochem. 27: 1424-1429.

Sternberger, L. A., P. H. Hardy, J. J. Curculis, and H. G. Meyer (1970) The unlabelled antibody-enzyme method of immunocytochemistry. Preparations and properties of soluble antigen-antibody complex (horseradish peroxidase-antihorseradish peroxidase) and its use in identification of spirochetes. J. Histochem. Cytochem. 18: 315-333.

Tago, H., H. Kimura, K. Kitahama, K. Sakai, M. Jouvet, and T. Maeda (1984) Cortical projections of monoamine oxidase-containing neurons from the posterior hypothalamus in the rat. Neurosci. I ett. 5?: 281-286

Taguchi, Y., T. Watanabe, H. Kubota, H. Hayashi, and H. Wada (1984) Purification of histidine decarboxylase from the liver of fetal rats and its immunochemical and immunohistochemical characterization. J. Biol. Chem. 259: 5214-5221.

Takeda, N., S. Inagaki, Y. Taguchi, M. Tohyama, T. Watanabe, and H. Wada (1984) Origins of histamine-containing fibers in the cerebral cortex of rats studied by immunohistochemistry with histamine decarboxylase as a marker and transection. Brain Res. 323: 55-63.

Takeda, N., S. Inagaki, S. Shiosaka, Y. Taguchi, W. H. Oertel, M. Tohyama, T. Watanabe, and H. Wada (1985) Immunohistochemical evidence for the coexistence of histidine decarboxylase-like and glutamate decarboxylaselike immunoreactivities in nerve cells of the magnocellular nucleus of the posterior hypothalamus of rats. Proc. Natl. Acad. Sci. U. S. A. 81: 76477650.

Tramu, G. A. Pillez, and J. Leonardelli (1978) An efficient method of antibody elution for successive or simultaneous localization of two antigens by immunocytochemistry. J. Histochem. Cytochem. 26: 322-324.

Ulmar, G., A. Ljungdahl, and T. Hökfelt (1975) Enzyme changes after undercutting of cerebral cortex in rat. Exp. Neurol. 46: 199-208.

Vincent, S. R., T. I lökfelt, and J. Y. Wu (1982) GABA neuron systems in hypothalamus and the pituitary gland: Immunohistochemical demonstration using antibodies against glutamate decarboxylase. Neuroendocrinology 34: $117-125$

Vincent, S. R., T. Hökfelt, L. R. Skirboll, and J. Y. Wu (1983) Hypothalamic $\gamma$-aminobutyric acid neurons project to the neocortex. Science 220: 13091311.

Watanabe, T., Y. Taguchi, H. Hayashi, J. Tanaka, S. Shiosaka, M. Tohyama $H$. Kubota, $Y$. Terano, and $H$. Wada (1983) Evidence for the presence of a histaminergic neuron system in the rat brain: An immunohistochemical analysis. Neurosci. Lett. 39: 249-254.

Watanabe, T., Y. Taguchi, S. Inagaki, J. Tanaka, H. Kubota, Y. Terano, M. Tohyama, and $\mathrm{H}$. Wada (1984) Distribution of the histaminergic neuron system in the central nervous system of rats; a fluorescent immunohisto. chemical analysis with histidine decarboxylase as a marker. Brain. Res. 295. 13-25.

Wilcox, B. J., and V. S. Seybold (1982) Localization of neuronal histamine in rat brain. Neurosci. Lett. 29: 105-110.

Wu, J. -Y. (1976) Purification and properties of L-glutamate decarboxylase (GAD) and GABA-aminotransferase (GABA-T). In GABA in Nervous System Function, E. Roberts, T. Chase, and D. Tower, eds., pp. 7-55, Raven Press, New York.

Wu, J. -Y., T. Matsuda, and E. Roberts (1973) Purification and characterization of glutamate decarboxylase from mouse brain. J. Biol. Chem. 248: 30293034 